\title{
Muscle hypertrophy after partial denervation: a human case
}

\author{
JAMES L. BERNAT, A N JOSE L. OCHOA \\ From the Division of Neurology, Department of Medicine, Dartmouth Medical School, Hanove1, \\ New Hampshire, USA
}

SUMMARY While undergoing long-term physiotherapy, a 41 year old woman with a chronic $\mathrm{S} 1$ radiculopathy developed progressive, painless enlargement of the weak calf. Gastrocnemius muscle biopsy disclosed changes of partial denervation and reinnervation, with small groups of type I and type II atrophic muscle fibres and abundant hypertrophic fibres of both types but mostly type II. It is postulated that, in addition to compensatory work-induced type II muscle fibre hypertrophy, there was an element of (type I) stretched-induced hypertrophy of denervated fibres, a condition well recognised experimentally but not documented in man.

Morpurgo (1897) first showed that muscle fibres will hypertrophy when subjected to increased work load. Sola and Martin (1953) showed that muscle fibre hypertrophy will occur with stretch, even in the absence of innervation. While muscle hypertrophy after nerve injury has been the subject of much experimental work, only rarely have human cases been documented. We present a patient who developed true muscle fibre hypertrophy in the setting of partial denervation and exercise, and propose that in this case the denervation predisposed to muscle hypertrophy from a combination of work and stretch.

\section{Case report}

A 41 year old woman was admitted to the Mary Hitchcock Memorial Hospital because of progressive right calf enlargement. Two years earlier she had developed low back pain which radiated into the right buttock and foot. Her physician found that she had hypalgesia over the lateral aspect of the right foot and absent right ankle reflex. Lumbosacral roentgenography disclosed narrowing of the L5-S1 disc space. She was treated with strict bed rest and the pain resolved, leaving only residual foot numbness. The pain recurred thereafter only while coughing, sneezing, and

Address for reprint requests: Dr J. L. Bernat, Division of Neurology, Department of Medicine, Dartmouth Medical School, Hanover, New Hampshire 03755, USA.

Accepted 18 February 1978 straining. The patient then started a vigorous exercise programme of walking and running which she continued regularly to the time of admission.

Nine months before admission she noted that her right calf was getting larger. This growth increased steadily and did not seem to be accompanied by similar growth in the left leg. She denied pain or tenderness of the enlarging extremity but noted a worsening of the old right buttock and foot pain and persistence of right foot numbness. There was no family history of neuromuscular disease, and the patient had no children.

Examination on admission showed her to be a moderately obese woman in excellent general health. There was no back tenderness or lumbar muscle spasm. Right straight leg raising to $60^{\circ}$ reproduced her buttock and foot pain. The right leg had normal colour, temperature, and arterial pulses. The circumference of the right calf, $150 \mathrm{~mm}$ distal to the patella, was $370 \mathrm{~mm}$ as compared to $335 \mathrm{~mm}$ on the left. There was no muscle tenderness or other abnormality to palpation, and the gastrocnemius idiomuscular response was normal. Muscle strength was normal except for trace weakness of the right extensor hallucis longus and moderate weakness of ankle plantar flexion demonstrable on repetitive toe standing. There was no myotonia. There was hypalgesia to pinprick over the right leg and foot as illustrated in Fig. 1. Deep tendon reflexes were normal except for an absent right ankle reflex. Plantar responses were flexor. 


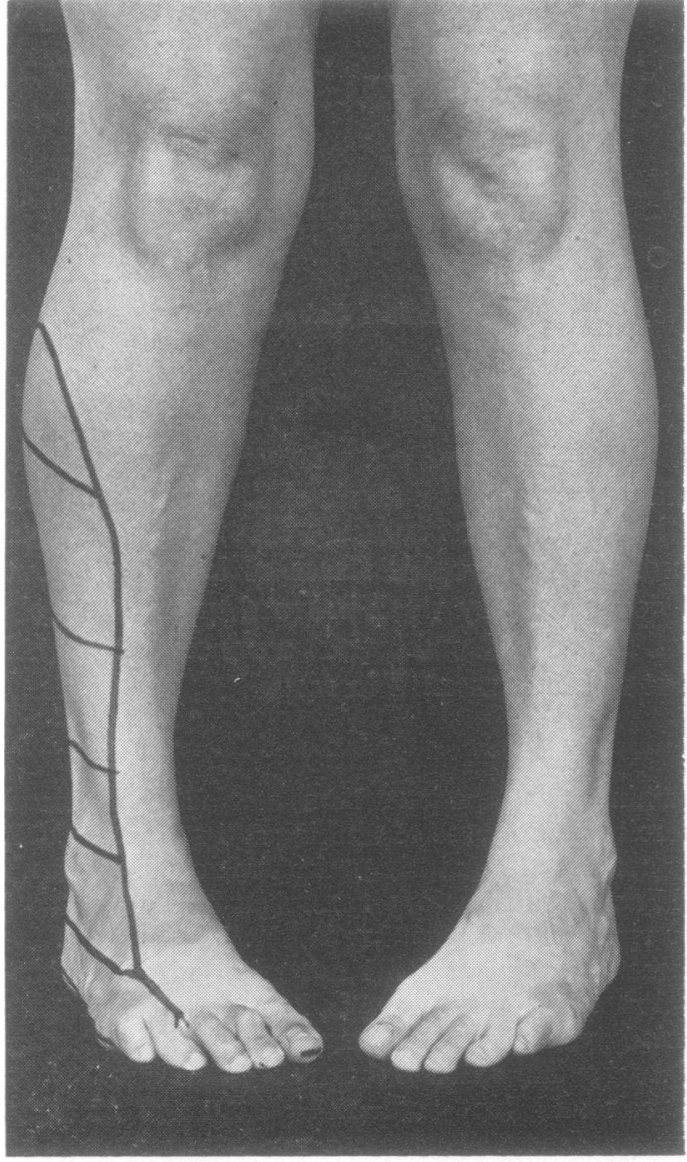

(a)

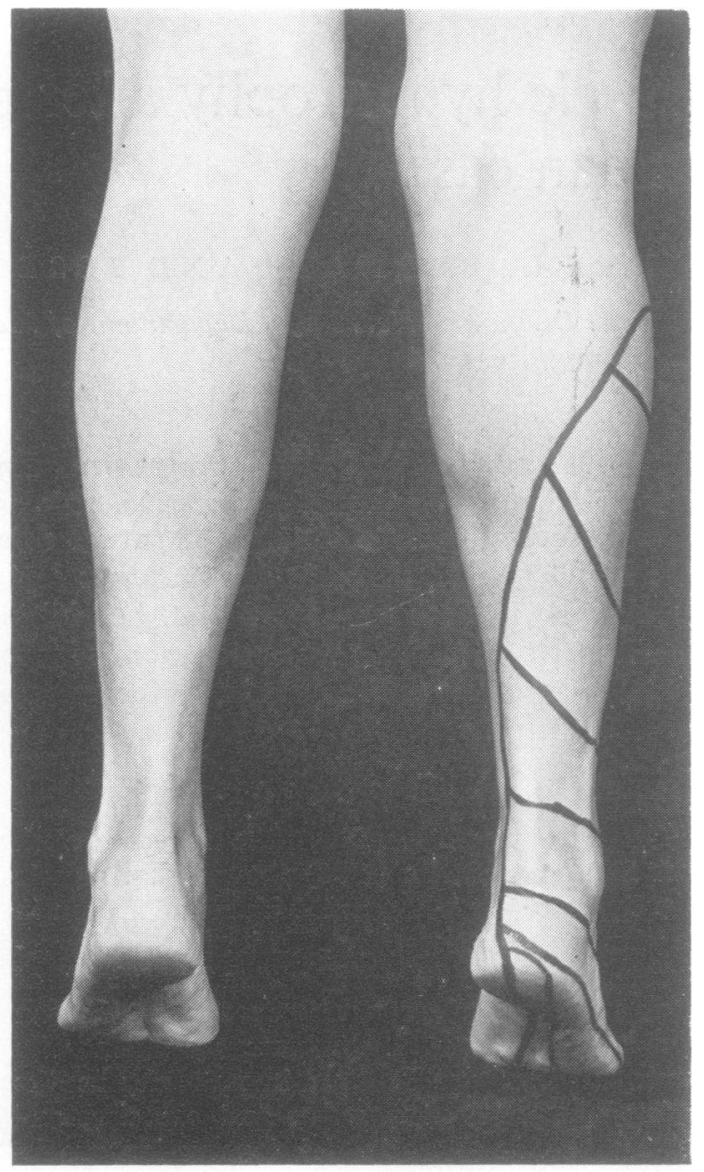

(b)

Fig. 1 Photographs of patient's legs revealing the enlarged right calf. The cross-hatched zone represents diminished sensation to pinprick.

Complete blood count was normal. Creatine phosphokinase (CPK) measured on two separate occasions was $126 \mathrm{IU} / 1$ and $85 \mathrm{IU} / 1$ (normal range 5-70 IU/1). Plain radiographs of the right calf failed to disclose a cystic cavity. Computed tomography (CT) scan of the right calf (Fig. 2) showed hypertrophy of the muscles of the posterior compartment. Electromyography (EMG) of the medial and lateral heads of the right gastrocnemius showed motor unit potentials up to $10 \mathrm{mV}$ in amplitude with a reduced voluntary pattern despite a normal firing rate. No myotonic discharges were present and there was no spontaneous electrical activity. Electromyograms of the right tibialis anterior and the right vastus medialis were normal.
Biopsy of the right gastrocnemius lateral head (Fig. 3) showed abnormal variation of muscle fibre size with atrophy and hypertrophy. There was atrophy, hypertrophy, and a tendency to type grouping of both type I and II fibres. There were many target fibres, a moderate number of central nuclei, and rare examples of fibre splitting and rimmed vacuoles. There was no infiltration with inflammatory cells, collagen, or fat. Hypertrophic fibres were abundant and occupied most of the volume of the muscle. These fibres reached 140 $\mu \mathrm{m}$ in diameter and were of both types, but predominantly type II. Atrophic fibres were also of both types, but predominantly type I. Upon subtyping muscle fibres of type II, it became apparent that subtypes IIA and IIB were equally hyper- 


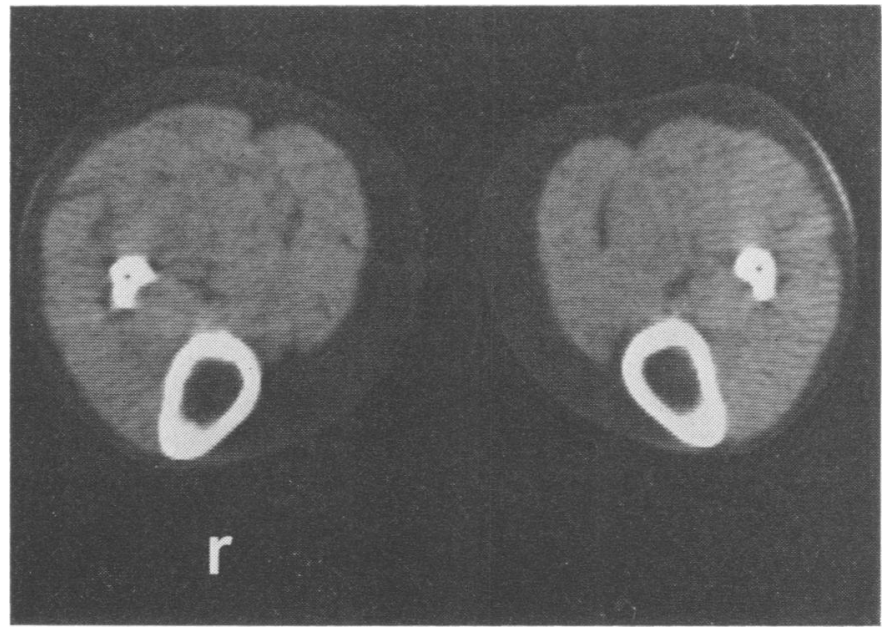

Fig. 2 Computed tomography scan taken transversely across the proximal third of the patient's legs. Enlargement on the right is due to the increased bulk of calf muscles.

trophic, whereas few IIA were atrophic. Due to the difficulty in the recognition of subtle colour changes in very small fibres, it was not possible to discern whether the type II atrophic fibres were of types IIB, IIC, or both. The size-frequency histogram for the fibre types is shown in Fig. 4.

Lumbosacral myelography disclosed a right sided extradural defect at the L5-S1 disc space with severe compression of the right first sacral nerve root.

At surgery, a calcified free fragment of the L5-S1 disc was identified adherent to and compressing the S1 nerve root. The scar tissue was divided with difficulty and the disc fragment was excised.

Postoperatively the patient was entirely free of pain and, when last examined four months after surgery, her ankle plantar flexion strength had improved objectively, and repeat measurements of calf girth were unchanged on the control side but were $10 \mathrm{~mm}$ less on the involved side. Electromyography was unchanged.

\section{Discussion}

A 41 year old woman developed painless enlargement of the right calf. Computed tomography scan showed this enlargement to be the result of muscle hypertrophy of the posterior compartment. Paradoxically, the enlarged muscles were weak and neurological and electromyographic examinations were diagnostic of a $\mathrm{S} 1$ radiculopathy. Surgery confirmed the myelographic findings of right posterolateral herniation of the L5-S1 intervertebral disc. Right gastrocnemius muscle biopsy revealed only evidence of partial denervation and reinnervation in addition to striking and rather type-selective muscle fibre atrophy and hypertrophy.

Enlargement of a muscle may result from an increase in the number or the size of muscle fibres (true hypertrophy) or from infiltration of the muscle by collagen, fat, parasites, tumour or inflammatory cells (pseudohypertrophy). True muscle hypertrophy occurs physiologically in workinduced hypertrophy, and pathologically in a variety of disorders including myotonia congenita, dystonias, acromegaly, and hypertrophia musculorum vera (Adams, 1975). Focal myositis and primary muscle neoplasm were initial diagnostic considerations in this case as the hypertrophy was painless and unilateral. The CT scan of the calf disclosed that the muscles of the right posterolateral compartment had normal density and configuration but were diffusely enlarged. This appearance was more consistent with true hypertrophy than with pseudohypertrophy or neoplasm, as muscle infiltration in the latter conditions usually produces an alteration in CT density (Weinberger and Levinsohn, 1978). Muscle neoplasm was felt to be an unlikely explanation as the hypertrophy was generalised and uniform in the affected muscles. The electromyographic findings indicated chronic partial denervation of both heads of the right gastrocnemius without similar involvement of neighbouring muscles innervated by nerve roots other than S1.

The muscle biopsy showed atrophy and grouping of both type I and II fibres, characteristic of chronic partial denervation and reinnervation. Some central nuclei were seen and there were occasional areas of fibre splitting. While these 

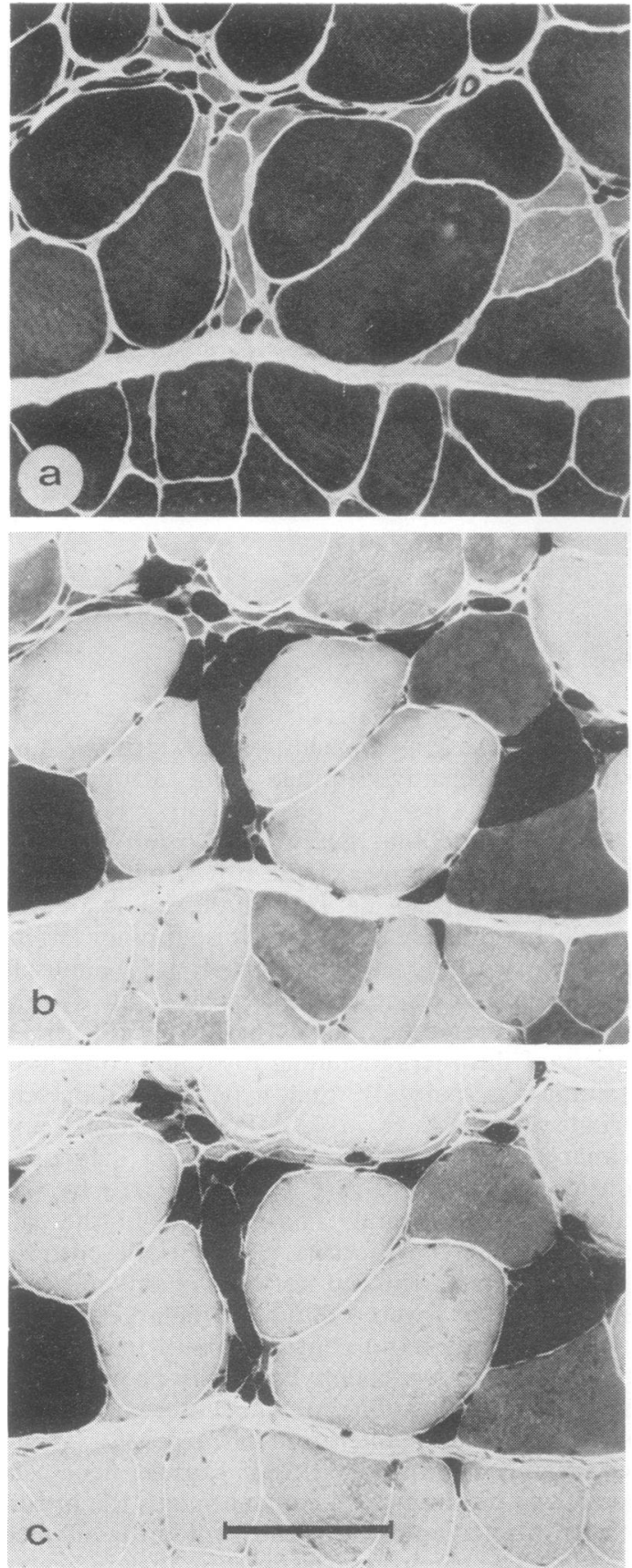

Fig. 3 Photomicrographs of biopsied right gastrocnemius muscle-a: routine ATPase at pH 9.4; $b$ : identical section with ATPase preincubated at pH 4.6; $c$ : identical section with ATPase preincubated at $\mathrm{pH}$ 4.3. These sections show four features:

hypertrophy predominantly involving type II fibres; atrophy predominantly involving type I fibres; type II fibre atrophy relatively sparing type IIA fibres;

Predominance of type II among the non-atrophic fibres. d: NADH-TR reaction shows several targets james L. Bernat, and Jose L. Ochoa
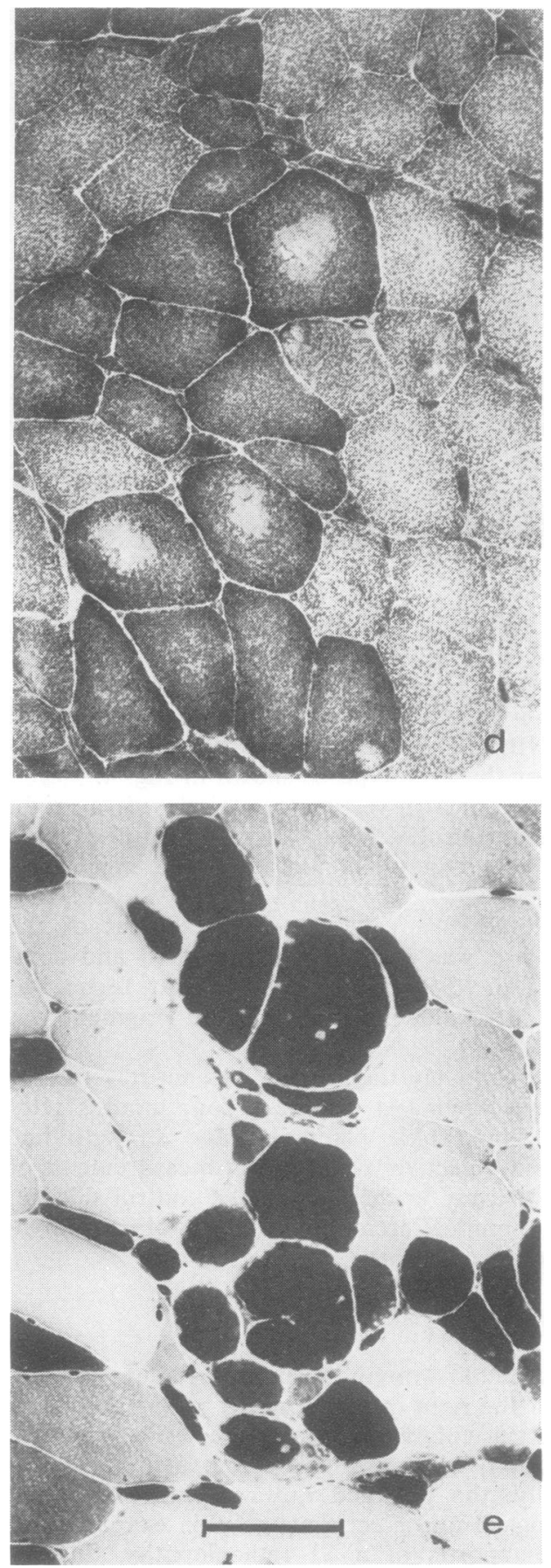

within grouped type I fibres; $e$ : ATPase at $\mathrm{pH} 4.6$ shows pseudomyopathic changes including central nuclei, fibre splitting, and fibre size variation without angular appearance. All bars represent $100 \mu \mathrm{m}$. 
latter findings generally suggest a myopathic disorder, they may be seen in partially denervated muscles (Drachman et al., 1967) and also in muscles subjected to increased work load (Hall-
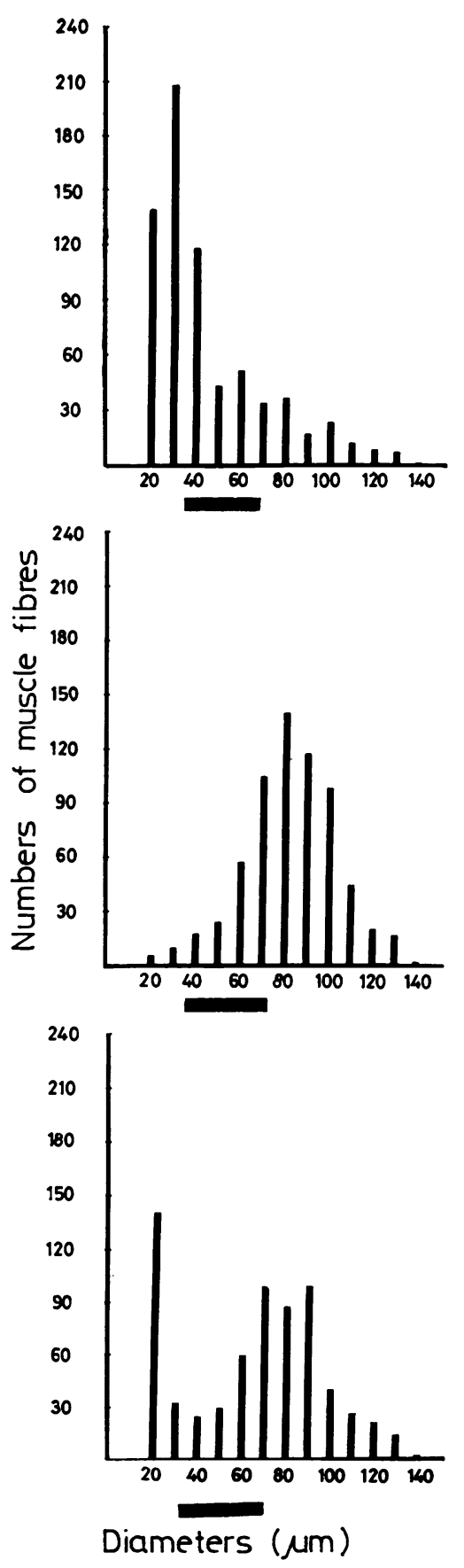

Craggs, 1972). The most remarkable finding in the muscle biopsy sample was the enormous increase in size of many muscle fibres, especially those of type II. Giant muscle fibres are known to result from an increased work load on the muscles (Denny-Brown, 1960; Goldspink and Howells, 1974). In work-induced hypertrophy, muscle fibres may exceed $100 \mu \mathrm{m}$ in diameter (Adams, 1968) and are usually type II (Dubowitz and Brooke, 1973). Thus, in the present case, the histological profile of the muscle and the history of strenuous leg exercise are consistent with the pathogenesis of work hypertrophy.

The acceptance of work hypertrophy as the sole pathogenesis of muscle enlargement leaves unanswered the issues of why the enlarged muscle was weak and why only the affected muscle enlarged, given equal exercise to the two legs. The paradox of muscle weakness despite increased muscle volume can be explained on the basis that a large number of fibres were atrophic, that a proportion of the hypertrophic fibres were denervated, and that the innervated hypertrophic fibres could not compensate for the denervated hypertrophic and atrophic fibres.

That only the partially denervated leg enlarged, despite equal exercise in the legs, suggests that chronic partial denervation somehow facilitates work hypertrophy. A possible explanation for this facilitation is that the work performed by the partially denervated muscle produced greater hypertrophy in the remaining innervated muscle fibres because there were fewer of them to perform the work than in the unaffected leg. Alternatively, there may have been a trophic influence from the partially denervated neuromuscular apparatus producing excess growth of the innervated muscle fibres.

While the model of work hypertrophy accounts for the type II fibre enlargement, the animal model of denervation hypertrophy may account for the type I fibre enlargement. Denervation-induced hypertrophy was first described as a transient event in the experimentally denervated rat diaphragm (Sola and Martin, 1953), and has since been described as a more permanent effect in ex-

Fig. 4 Muscle fibre diameter spectra based upon ATPase reactions. Top: type I fibres; middle: type IIA fibres; bottom: type IIB and IIC fibres. The bar under each histogram represents the range of normal muscle fibre diameters for the adult female (based upon Dubowitz and Brooke, 1973, Fig. 4.2, page 76). Note that most of the hypertrophic fibres are type II, more of the atrophic fibres are type I, very few type IIA fibres are atrophic, and that the atrophic type II fibres are smaller than the atrophic type I fibres. 
perimentally denervated muscle preparations of a variety of other species (Stewart et al., 1972; Sola et al., 1973; Asmussen and Kiessling, 1975, 1976; Sarnat et al., 1977). Sola et al. (1973) demonstrated that a denervated muscle subjected to constant stretch undergoes hypertrophy. They also found that a normally innervated muscle subjected to the same stretch undergoes even greater hypertrophy. They concluded that a muscle hypertrophies in proportion to the degree of stretch imposed on it, and that denervation does not necessarily predispose to hypertrophy if the stretch remains constant. Experimental models of denervation hypertrophy in the chicken, frog, and pigeon have shown that the hypertrophic fibres are usually type I (Cherian et al., 1966; Jirmanova and Zelena, 1970; Sarnat et al., 1977).

We propose that the type I fibre hypertrophy seen here was produced by increased stretching of the weak partially denervated muscle by its stronger antagonists during exercise. It is possible that a proportion of hypertrophic 2 fibres were similarly produced.

This case is consistent in some respects with cases that in the past have been termed hypertrophia musculorum vera. However, the entity hypertrophia musculorum vera does not have a unique pathology and appears to be a general designation for cases of acquired muscle enlargement caused by muscle fibre hypertrophy of diverse aetiologies. In this sense, the current case could be considered hypertrophia musculorum vera resulting from denervation and exercise.

There are only a few previous reports of patients with muscle hypertrophy after nerve injury. Graves (1848) reported a man with sciatica and subsequent leg enlargement. L'Hermitte (1918) reported a man with a bullet injury to the sciatic nerve and subsequent calf enlargement. McComas (1977) reported a woman with calf enlargement after analgesic injections into the sciatic nerve. The only previously reported case with muscle biopsy was that of Lapresle et al. (1973). They reported a man with a L4-5 herniated intervertebral disc with subsequent calf myotonia and hypertrophy. Gastrocnemius muscle biopsy, as in our case, revealed muscle fibre hypertrophy without increase in collagen. In contrast to our case, there was selective type I hypertrophy and type II atrophy, which closely approximate the experimental model of stretch-induced denervation hypertrophy but which the authors explained as denervation atrophy and compensatory hypertrophy. Although type specific atrophy is not generally regarded as a feature of denervation, the presence of target fibres and a known nerve injury does support a primarily denervating process.

The unifying principle in the two reported cases of biopsy proved true muscle hypertrophy after nerve injury is that the muscles were repeatedly stretched. In the current case, repeated muscle stretching and work were induced by strenuous exercise of the partially denervated limb. Although a history of exercise was not present in the case of Lapresle et al., myotonia may have accounted for repeated stretching of the partially denervated muscles. It seems reasonable to conclude that muscle hypertrophy is either work hypertrophy induced by repeated muscle contraction or stretch hypertrophy induced passively. Partial denervation may predispose to hypertrophy when some innervated fibres are overworked or when some innervated or denervated fibres are stretched.

We are grateful to Miss Elizabeth Boogusch for invaluable help with the muscle fibre counts.

\section{References}

Adams, R. D. (1968). The giant muscle fiber: its place 0 in myopathology. In Modern Neurology: Papers inō Tribute to D. Denny-Brown, pp. 225-240. Edited by的 S. Locke. Little, Brown, and Company: Boston. $\frac{\mathrm{P}}{\mathrm{D}}$ 읭 Adams, R. D. (1975). Diseases of Muscle. A Study in Pathology, Third Edition. Harper and Row: Hagers $\square$ town, Maryland.

Asmussen, G., and Kiessling, A. (1975). Hypertrophy응 and atrophy of mammalian extraocular muscle fibres following denervation. Experientia, 31, 11861188 .

Asmussen, G., and Kiessling, A. (1976). Kaliberveränderungen der muskelfasertypen des musculus obliquus inferior oculi des kaninchens nach denervierung. Acta Anatomica, 96, 386-403.

Cherian, K. M., Bokdawala, F. D., Vallyathan, N. V., and George, J. C. (1966). Effects of denervation on the red and white fibres of the pectoralis muscle of the pigeon. Journal of Neurology, Neurosurgery, and Psychiatry, 29, 299-309.

Denny-Brown, D. (1960). Experimental studies pertaining to hypertrophy, regeneration and degeneration. Research Publications: Association for Research in Nervous and Mental Disease, 38, 147-196. Williams and Wilkins: Baltimore.

Drachman, D. B., Murphy, S. R., Nigam, M. P., and Hills, J. R. (1967). "Myopathic" changes in chronically denervated muscle. Archives of Neurology (Chicago), 16, 14-24.

Dubowitz, V., and Brooke, M. H. (1973). Muscle Biopsy: A Modern Approach. W. B. Saunders and Company: London.

Goldspink, G., and Howells, K. F. (1974). Work induced hypertrophy in exercised normal muscles of different ages and the reversibility of hypertrophy after cessation of exercise. Journal of Physiology, 239, 179-193. 
Graves, R. J. (1848). Clinical Lectures on the Practice of Medicine. Second Edition Vol. 1, p. 498. Fannin and Company: Dublin.

Hall-Craggs, E. C. B. (1972). The significance of longitudinal fibre division in skeletal muscle. Journal of the Neurological Sciences, 15, 27-33.

Jirmanova, I., and Zelena, J. (1970). Effect of denervation and tenotomy on slow and fast muscles of the chicken. Zeitschrift für Zellforschung und Mikroskopische Anatomie, 106, 333-347.

Lapresle, J., Fardeau, M., and Said, G. (1973). L'hypertrophie musculaire vraie secondaire à une atteinte nerveuse périphérique: étude clinique et histologique d'une observation d'hypertrophie du mollet consécutive à une sciatique. Revue Neurologique, 128, 153-160.

L'Hermitte, J. (1918). Hypertrophie des muscles de la jambe consécutive à une lésion du nerf sciatique par balle. Revue Neurologique, 25, 56-58.

McComas, A. J. (1977). Neuromuscular Function and Disorders. Butterworths: London.

Morpurgo, B. (1897). Ueber Activitats-Hypertrophie der Willkurlichen Muskeln. Virchows Archiv für
Pathologische Anatomie und Physiologie, 150, 522554.

Sarnat, H. B., Portnoy, J. M., and Chi, D. Y. K. (1977). Effects of denervation and tenotomy on the gastrocnemius muscle in the frog: a histologic and histochemical study. Anatomical Record, 187, 335346.

Sola, O. M., and Martin, A. W. (1953). Denervation hypertrophy and atrophy of the hemidiaphragm of the rat. American Journal of Physiology, 172, 324332.

Sola, O. M., Christensen, D. L., and Martin, A. W. (1973). Hypertrophy and hyperplasia of adult chicken anterior latissimus dorsi muscles following stretch with and without denervation. Experimental Neurology, 41, 76-100.

Stewart, D. M., Sola, O. M., and Martin, A. W. (1972). Hypertrophy as a response to denervation in skeletal muscle. Zeitschrift für Vergleichende Physiologie, 76, 146-167.

Weinberger, G., and Levinsohn, E. M. (1978). Computed tomography in the evaluation of sarcomatous tumours of the thigh. American Journal of Roentgenology, 130, 115-118. 